\title{
Novel alkaloids from the fire ant, Solenopsis geminata
}

\author{
Robert K. Vander Meer ${ }^{1}$. Satya P. Chinta ${ }^{2}$. Tappey H. Jones ${ }^{3}$
}

Received: 24 September 2021 / Revised: 6 January 2022 / Accepted: 13 January 2022 / Published online: 27 January 2022

This is a U.S. government work and not under copyright protection in the U.S.; foreign copyright protection may apply 2022

\begin{abstract}
South American fire ants, Solenopsis richteri and Solenopsis invicta, were accidently introduced into the southern USA in the 1900s and 1930s, respectively. The rapid spread and high population densities of S. invicta, and its potent sting, resulted in broad economic impacts and a variety of research efforts. In the 1970s, their venom alkaloids were identified as a complex blend of trans-2-methyl-6-alkyl- and alkenyl-piperidines. Solenopsis geminata is a worldwide tramp species but a native of the southern coastal regions of the USA. It was found to only produce $c i s$ - and trans-2-methyl-6-undecyl-piperidines. These alkaloids were considered the Solenopsis ancestral alkaloid profile since they were identified from female sexuals (potential queens) of all Solenopsis species in South and North America. The dramatic modification of alkaloids in Solenopsis invicta was attributed to their response to evolutionary pressure and the lack of change in $S$. geminata alkaloids due to no response to evolutionary pressure. Here we report the unexpected discovery of 6-undecyl-pyridine, 2-methyl-6-undecyl-pyridine and 2-methyl-6-(1)-undecenyl-pyridine as components of $S$. geminata worker venom, suggesting that $S$. geminata like its South American relatives have responded to evolutionary pressures. Our results will stimulate future research on S. geminata populations throughout the tropical/subtropical world.
\end{abstract}

Keywords Solenopsis geminata $\cdot$ Alkaloids $\cdot$ Fire ant $\cdot$ Venom $\cdot$ Piperidine $\cdot$ Pyridine

\section{Introduction}

The fire ants, Solenopsis geminata and Solenopsis xyloni (members of the $S$. geminata species complex, Pitts et al. 2018), are native to the southern USA and have a long history of human interactions. While they have a significant sting, it was the accidental introduction of two South American fire ants into the southern USA, Solenopsis richteri (1920s) and especially Solenopsis invicta (1930s) (Solenopsis saevissima species complex, Pitts et al. 2018), which generated widespread attention. High population densities of Solenopsis invicta and a propensity for disturbed habitats led

Communicated by: John A. Byers

Robert K. Vander Meer

bob.vandermeer@usda.gov

1 USDA/ARS, CMAVE, 1600 SW 23rd Drive, Gainesville, FL 32608, USA

2 Foresight Science and Technology, Hopkinton, MA 01748, USA

3 Department of Chemistry, Virginia Military Institute, Lexington, VA 24450, USA to frequent human interactions and broad economic impact. Five trans-2-methyl-6-alkyl- $\left(\mathrm{C}_{11: 0}, \mathrm{C}_{13: 0}, \mathrm{C}_{15: 0}\right)$ and alkenyl- $\left(\mathrm{C}_{13: 1}, \mathrm{C}_{15: 1}\right)$-piperidines were identified from $S$. invicta worker venom (MacConnell et al. 1971). Venom alkaloid research was extended to workers of the native fire ants, $S$. geminata and S. xyloni. They produce a mixture of cis- and trans-2-methyl-6-undecyl-piperidines (Brand et al. 1972, Fig. 1, compounds 2 and 4), in contrast to the variety of piperidine alkaloids that dominate the two invasive species. Since all female sexual forms (future queens) associated with the $S$. geminata and S. saevissima species complexes produce cis- and trans-2-methyl-6-undecyl-piperidines, these two alkaloids were considered the ancestral type. It was thought $S$. invicta and $S$. richteri workers evolved beyond the undecyl-piperidines, while $S$. geminata species complex workers maintained the ancestral alkaloid blend (Brand, 1978).

Alkaloid use in chemotaxonomy was illustrated by Brand et al. (1973), in that S. xyloni and S. geminata workers could be identified by their cis/trans 2-methyl6-undecyl-piperidine ratio. A broad survey of Solenopsis venom alkaloids in North and South America also supported alkaloid use in chemotaxonomy (MacConnell et al. 
Fig. 1 The total ion chromatogram is shown for an extract of $S$. geminata workers (Gainesville, FL) immersed in hexane for $24 \mathrm{~h}$. The seven peaks were demonstrated to be composed of single components rather than mixtures of co-eluting compounds (MassWorks, Cerno Bioscience, https://cernobiosc ience.com). The structures for six of the seven alkaloids were determined by mass spectral fragmentation patterns, synthesis, and co-injection and are inserted into the chromatogram

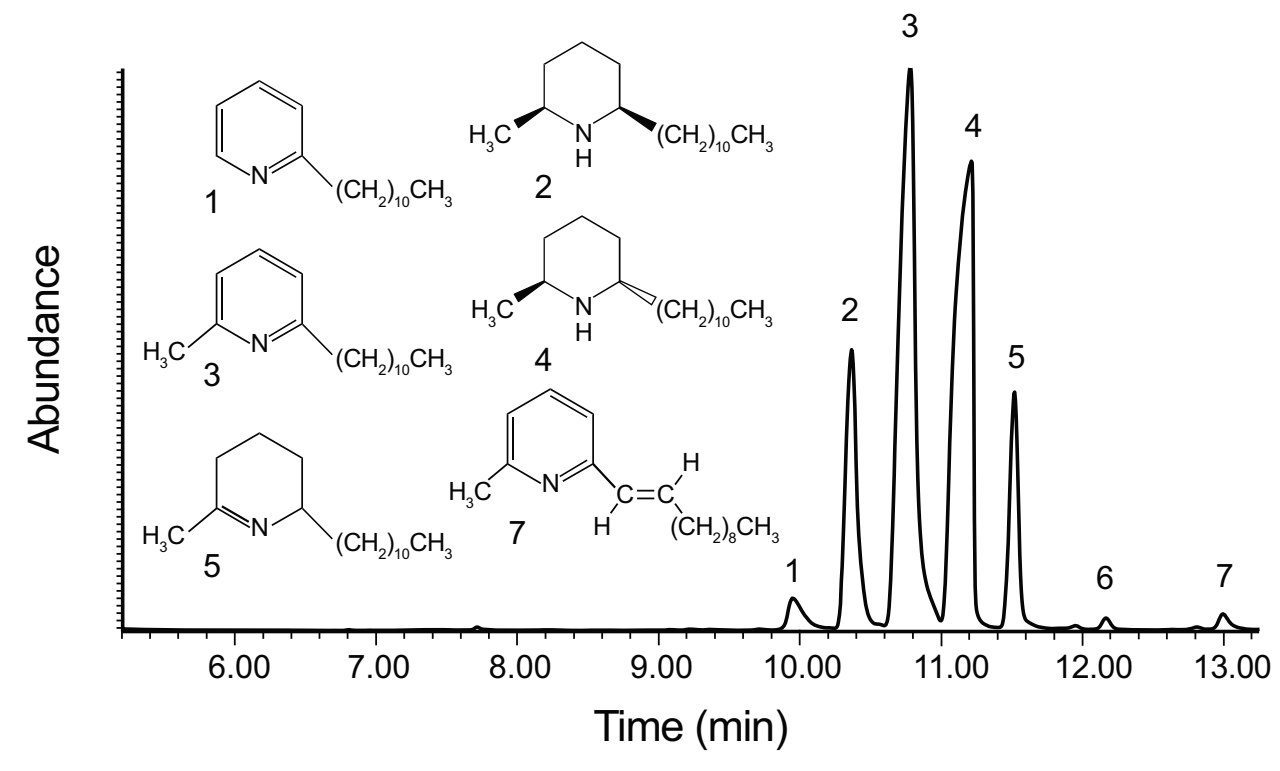

1976). Also, Brand et al. (1972) found detectable amounts of 2-methyl-6-undecyl- $\Delta^{1,2}$-piperideine (see Fig. 1, compound (5), in S. xyloni venom) but suggested it was a precursor to the major piperidine venom components, as later verified by Leclercq et al. (1996). A reproductively viable and morphologically cryptic hybrid between S. invicta and $S$. richteri was initially detected based on their venom alkaloid profiles (Vander Meer and Lofgren, 1985).

Piperideine alkaloids from $S$. invicta were reported as minor components by two research groups (Chen et al. 2009; Chen and Fadamiro, 2009). Both groups suggested that the piperideines likely functioned as piperidine precursors (Leclercq et al. 1996). A focused study of $S$. geminata venom alkaloids (Shi et al. 2015) isolated the expected cis- and trans-2-methyl-6-undecyl-piperidines as the major components. Pyridine alkaloids were not reported from Solenopsis venom until Chen et al. (2019) described trace amounts of ten pyridines from workers of S. invicta, S. richteri, and their hybrid. The identified pyridines were each $<1 \mathrm{ng} / \mathrm{ant}$ in both species and their hybrid. For perspective, each $S$. invicta worker produces about $18 \mu \mathrm{g}$ of venom alkaloids (Haight and Tschinkel 2003).

Solenopsis geminata alkaloids were well established as cis-and trans-2-methyl-6-undecyl-piperidines when in 2019 three worker ants intercepted at a port in Hawaii, identified as Solenopsis, were sent to our facility to determine if they were $S$. invicta. Molecular analyses (mitochondrial $C O X 1$ gene) classified the ants as $S$. geminata. Chemical analysis unambiguously identified two major components as 2-methyl-6-undecyl-pyridine and trans2-methyl-6-undecyl-piperidine. The normally dominant cis-2-methyl-6-undecyl-piperidine was a minor component (Ascunce et. al. 2021). This surprising result was the driving force for reinvestigation of $S$. geminata venom alkaloids.

Here we report the unexpected discovery of 6-undecylpyridine, 2-methyl-6-undecyl-pyridine, and 2-methyl-6-(1)undecenyl-pyridine (compounds 1, 3, and 7 in Fig. 1) as new alkaloid components of $S$. geminata venom from Gainesville, FL, USA.

\section{Methods}

\section{Sampling}

The tropical fire ant, $S$. geminata, inhabits the southern tier of the USA and can be found in the Gainesville, FL, area, normally in dry, well-drained areas that are less likely to be occupied by the invasive fire ant, S. invicta. Solenopsis geminata is unique in that it can be readily differentiated from other Solenopsis species by the presence of a worker caste that has a disproportionately large head. All samples of $S$. geminata workers (20-50) were collected from individual colonies (replicates) and placed in a vial containing enough hexane (Fisher Optiva, Cole Palmer, Vernon Hills, IL) to cover the ants. After $24 \mathrm{~h}$, the hexane was pipetted into a clean vial in preparation for GC-MS analysis to determine tentative component identification and relative component percentages. Collection site A was sampled in 2015. At that time, cuticular hydrocarbon patterns were of prime interest since the alkaloids were assumed to be $\mathrm{cis}$ and trans-2-methyl-6-undecylpiperidines. The GC-MS temperature program targeted separation of cuticular hydrocarbons. The two major alkaloid peaks (2) and (4), Fig. 1, matched the expected cis- and trans-piperidine fragmentation pattern (base fragment at $m / e=98$, see Fig. 2). 
Fig. 2 The mass spectral fragmentation patterns and associated structures are shown for each of the 6 identified $S$. geminata alkaloid components from an extract of $S$. geminata workers (Gainesville, FL) immersed in hexane for $24 \mathrm{~h}$. The structures for six of the seven alkaloids were determined by mass spectral fragmentation patterns, synthesis, and co-injection
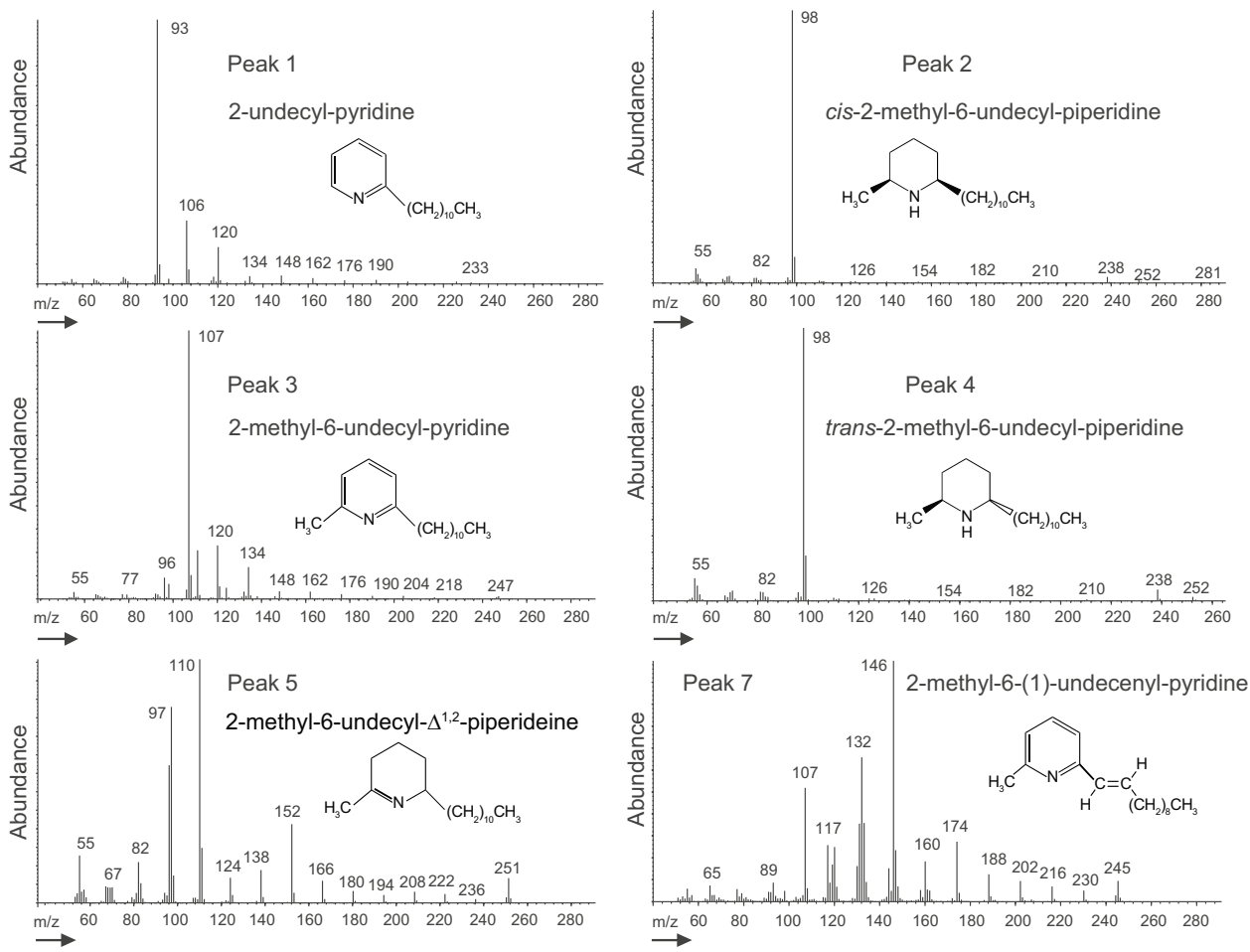

The alkaloids are antibiotic and very stable and have been recovered after decades of room temperature storage (see method for synthetic component 3). In view of the Hawaii results (Ascunce et al., 2021), the 2015 samples (site A, $n=9$ ) were reconstituted in hexane, vortexed, and analyzed using GC-MS program b. Fragmentation patterns (Fig. 2) were compared to synthetic standards. Components 1 to 5 and 7 (Fig. 1) were identified. The TIC (total ion chromatogram) was used to integrate the areas under each peak, and the peak percent composition was calculated. Twelve additional samples were collected and analyzed in 2019 from Gainesville, FL, site B, which was approximately
$25 \mathrm{~km}$ from site A. Graphical representations of alkaloid peak proportions were prepared (Fig. 3, site A and site B) using GraphPad Prism, version 9.01 (GraphPad Software Inc., San Diego, CA).

\section{Gas chromatograph-mass spectroscopy (GC-MS) analysis}

Mass spectral data were obtained from (a) Shimadzu QP-2020 GC-MS (Palo Alto, CA) equipped with an RTX-5 ms, $30 \mathrm{~m} \times 0.25 \mathrm{~mm}$ i.d. column. The instrument was temperature programmed from 60 to $250^{\circ} \mathrm{C}$ at $10^{\circ} /$ min and
Fig. 3 The mean + SE of the percent composition for the 7 alkaloid components based on the integration values for each sample (total ion chromatogram, TIC: (1) collection site A, 2015, $n=9$, and (2) collection site B, 2019. Locations A and B are approximately $25 \mathrm{~km}$ apart. Each replicate represents a unique $S$. geminata colony
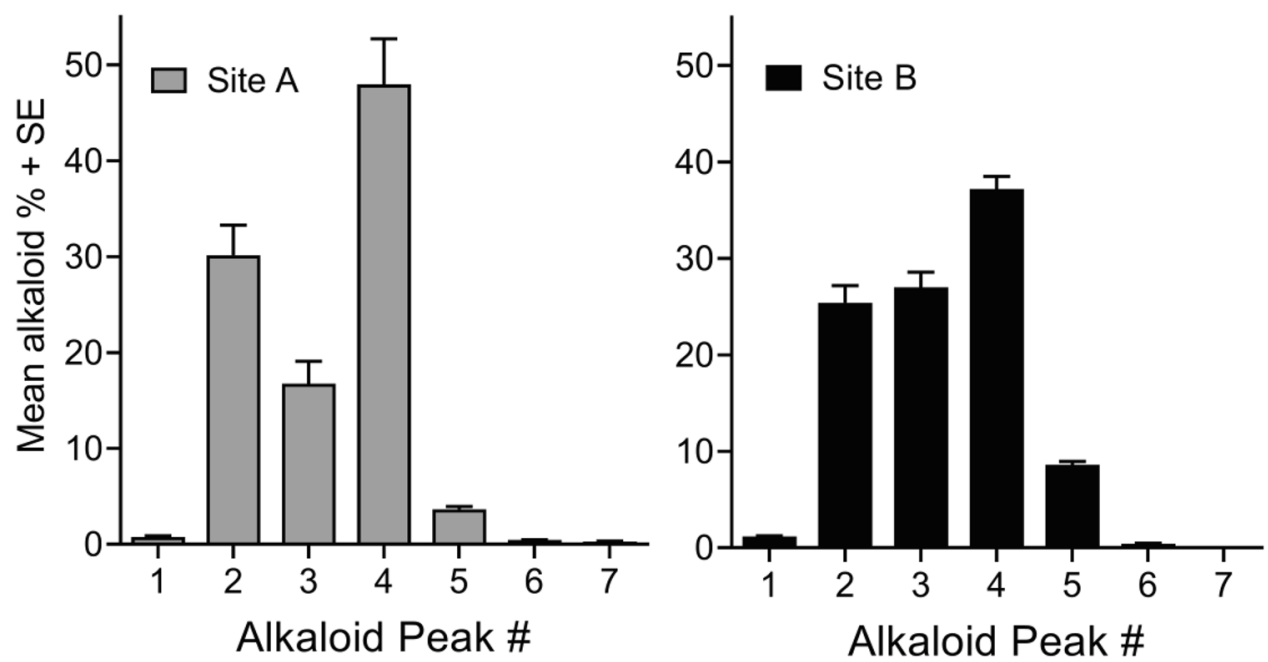
held there for 20 min (Virginia Military Institute, all synthetic samples) or (b) Agilent Intuvo 9000 GC system (Santa Clara, CA) equipped with an HP-5 MS ultra-inert nonpolar column, $30 \mathrm{~m} \times 0.25 \mathrm{~m}$ i.d. column, coupled to a $5977 \mathrm{~B}$ mass spectral detector and a MassHunter Data Acquisition Workstation version 10.0.368 (Santa Clara, CA). Injector temperature was set at $250{ }^{\circ} \mathrm{C}$. The oven temperature was programmed at $40{ }^{\circ} \mathrm{C}$ for $2 \mathrm{~min}$ and then to $285^{\circ} \mathrm{C}$ at $5{ }^{\circ} \mathrm{C} /$ min, followed by a $10 \mathrm{~min}$ hold at $285^{\circ} \mathrm{C}$ (USDA, Gainesville, Florida). Agilent's MassHunter and NIST MS Library Database (2020) was used to compare venom component mass spectral fragmentation signatures (see Fig. 2). MassWorks software (Cerno Bioscience, https://cernobioscience. $\mathrm{com} /$ ) determined the purity of each peak and their retention index (RI) relative to a series of normal hydrocarbons.

\section{Results}

\section{Identification of compounds 1-5 and 7}

See Fig. 2 for the mass spectral fragmentation pattern of each compound.

\section{2-undecyl-pyridine (1)}

Venom component 1 (Fig. 1) was tentatively identified as 2-undecyl-pyridine through a search and match of the NIST (2020) mass spectrum library. To verify the structure assignment, the known compound was synthesized by alkylation of 2-picolyl magnesium bromide with 1-bromodecane (Proffe and Linke, 1960). The synthetic product was distilled by Kugelrohr distillation and had a mass spectral fragmentation pattern that matched the reported fragmentation pattern (Heller et al. 2002) for 2-undecyl-pyridine (1). This pattern was identical to that of natural $S$. geminata component (1). In addition, synthetic 2-undecyl-pyridine co-eluted with natural component (1) when co-injected on the GC-MS, verifying the structural assignment.

\section{cis-2-methyl-6-undecyl-piperidine (2), 2-methyl-6-undecyl-pyridine (3), and trans-2-methyl-6-undecyl-piperidine (4)}

These venom components are known compounds and were synthesized by methods described in MacConnell et al. (1971). Component (3) was distilled from an original 50-year-old sample synthesized by MacConnell et al. (1971), a testament to the stability of the pyridines. GC-MS retention times, co-injections, and matched fragmentation patterns confirmed the assigned structures of venom components, (2), (3), and (4).

\section{2-methyl-6-undecyl- $\Delta^{1,2}$-piperideine (5)}

Component (5) was synthesized by the method described by Brand et al. (1972). The known fragmentation pattern for compound (5) and the other piperideine isomer, 2-methyl-6undecyl- $\Delta^{1,6}$-piperideine, are distinctive (Brand et al. 1972). The fragmentation pattern of $S$. geminata component (5), Fig. 2, matched the fragmentation pattern of the 2-methyl6 -undecyl- $\Delta^{1,2}$-piperideine (5). Synthetic component (5) coinjected with the natural extract, co-eluted with tentatively identified component 5 , confirming its structure.

\section{2-methyl-6-(1)-undecenyl-pyridine (7)}

Proposed component (7) (see Fig. 1) is a known compound and was prepared as described by Okuma et al. (2003) via a Wittig reaction of decyltriphenylphosphonium bromide with 6-methyl-2-pyridine-carboxyaldehyde, using DBU (1,8-diazabicyclo-[5.4.0]-undec-7-ene) as a base. The products of this reaction were a $1: 1$ mixture of $Z$ - and $E$-2-methyl-6-(1)-undecenyl-pyridine. The mass spectrum (see Fig. 2) and retention time of the trans- $(E)$ isomer matched those of component (7) from $S$. geminata. Co-injected $E$-2-methyl-6-(1)-undecenyl-pyridine co-eluted with component (7) in a natural $S$. geminata sample, confirming the structural assignment.

The retention index (RI) was calculated for the seven alkaloid components (Fig. 1) via MassWorks (Cerno Bioscience, https://cernobioscience.com): $(1)=1815$, $(2)=1835,(3)=1854,(4)=1874,(5)=1891,(6)=1928$, and $(7)=1959$. The first five alkaloids elute in between $\mathrm{C}_{18}$ and $\mathrm{C}_{19}$ normal hydrocarbons.

The GC-MS data provided tentative identification of Fig. 1 peaks: (1) 2-undecyl-pyridine, (2) cis-2-methyl6-undecyl-piperidene, (3) 2-methyl-6-undecyl-pyridine, (4) trans-2-methyl-6-undecyl-piperidene, (5) 2-methyl-6undecyl- $\Delta^{1,2}$-piperideine, and (7) 2-methyl-6-(1)-undecenylpyridine. Peaks (2) and (4) are the cis- and trans-disubstituted major components reported early on for $S$. geminata (see "Introduction"). Component 5 was previously reported as minor components from $S$. geminata. Alkaloid components (1), (3), and (7) are known compounds but are reported here for the first time from Solenopsis geminata. Components (1) and (7) are reported for the first time from Solenopsis ants. Component 6 had a molecular ion at $M+=263$, and its mass spectrum suggested an N, 2-dimethyl-6-undecyldihydropyridine. The small amount present relative to the other alkaloids prevented further characterization. The relative abundance (mean + SE) of the 7 alkaloid components (Fig. 1) from collection site A, $n=9$, and site $\mathrm{B}, n=12$, is shown in Fig. 3. Replicates represent $S$. geminata worker samples collected from different colonies located at the collection sites. 


\section{Discussion}

The driving force for this research was the unexpected identification of 2-methyl-6-undecyl-pyridine, Fig. 1, peak (3) as the major component from three $S$. geminata ants intercepted by the State of Hawaii quarantine officials (Ascunce et al., 2021). This discovery was contrary to the literature that over four decades reinforced cis- and trans-2-methyl-6-undecylpiperidines as the major $S$. geminata venom alkaloids (Brand et al. 1972 to Shi et al. 2015). Prior to the 1980s, packed columns were not capable of separating the pyridines and piperidines reported here, e.g., MacConnell et al. (1976). Lai et al. (2009) investigated seasonal and caste changes in S. geminata worker venom from hexane extracts analyzed via GC-MS with a capillary column. They used a very short temperature program ( $8 \mathrm{~min}$ ) but used single ion monitoring (SIM), $\mathrm{m} / \mathrm{z} 98$, to generate data specific to the piperidines (see Fig. 2). The pyridines and piperideines do not have an m/z 98 fragment (Fig. 2) and would be excluded. Shi et al. (2015) applied a special silica gel gravity column separation method to isolate piperidines from a total $S$. geminata extract (from Gainesville, FL) prior to GC-MS analysis. They did not identify any pyridines. Our slow GC temperature ramp led to baseline separation and identification of the six alkaloids presented here.

Our results increase the complexity of $S$. geminata alkaloids, suggesting that $S$. geminata workers have indeed responded to evolutionary pressure with alkaloid diversity more complicated than the ancestral cis/trans 2-methyl-6-undecyl-piperidines found in Solenopsis female sexuals. What makes our results especially intriguing is that $S$. geminata occupied coastal areas of the southern USA, and with the advent of worldwide trade routes in the mid-sixteenth century, S. geminata accidentally hitchhiked to many tropical/ subtropical areas of the world (Wetterer 2011; Gotzek et al. 2015). The ecological habitats that $S$. geminata found itself in and survived in are extraordinarily diverse, e.g., Senegal, Canary Islands, Madagascar, Australia, Fiji, Gilbert Islands, India, Laos, and Italy (Wetterer 2011). The specific focus for this paper is on S. geminata in the USA and specifically Florida, but the results will be of interest to researchers throughout the tropical/subtropical areas of the world where S. geminata is found.

Acknowledgements The authors would like to thank Erin O'Reilly, Andrew Nisip, and Michele Nitti for technical support.

Author contribution All authors contributed equally.

Data availability The data sets generated during and/or analyzed during the current study are available from the corresponding author on reasonable request.

Code availability NA.

\section{Declarations}

Ethics approval NA

Consent to participate NA

Consent for publication NA

Conflict of interest The authors declare no competing interests.

Open Access This article is licensed under a Creative Commons Attribution 4.0 International License, which permits use, sharing, adaptation, distribution and reproduction in any medium or format, as long as you give appropriate credit to the original author(s) and the source, provide a link to the Creative Commons licence, and indicate if changes were made. The images or other third party material in this article are included in the article's Creative Commons licence, unless indicated otherwise in a credit line to the material. If material is not included in the article's Creative Commons licence and your intended use is not permitted by statutory regulation or exceeds the permitted use, you will need to obtain permission directly from the copyright holder. To view a copy of this licence, visit http://creativecommons.org/licenses/by/4.0/.

\section{References}

Ascunce MS, Vander Meer RK, Chinta SP, Ogura-Yamada C, and Oishi D (2021). Genetic and chemical profiling of fire ants intercepted in Hawaii. The Florida Entomologist

Brand et al (1973) Biochemical evolution in fire ant venoms. Insect Biochem 3(9):45-51. https://doi.org/10.1016/0020-1790(73) 90017-6

Brand JM (1978) Fire ant venom alkaloids: their contribution to chemosystematics and biochemical evolution. Biochem Syst Ecol 6:337-340. https://doi.org/10.1016/0305-1978(78)90055-8

Brand JM, Blum MS et al (1972) Fire ant venoms: comparative analyses of alkaloidal components. Toxicon 10(3):259-271. https://doi. org/10.1016/0041-0101(72)90011-6

Chen L, Fadamiro HY (2009) Re-investigation of venom chemistry of Solenopsis fire ants. I. Identification of novel alkaloids in S. richteri. Toxicon 53(5):469-478

Chen J, Cantrell CL et al (2009) Piperideine alkaloids from the poison gland of the red imported fire ant (Hymenoptera: Formicidae). J Agric Food Chem 57(8):3128-3133. https://doi.org/10.1021/ jf803561y

Chen J, Zhao Y, Li X-C, Zhao J-H (2019) Pyridine alkaloids in the venom of imported fire ants. J Agric Food Chem 67:1138811395. https://doi.org/10.1021/acs.jafc.9b03631

Gotzek D, Axen HJ et al (2015) Global invasion history of the tropical fire ant: a stowaway on the first global trade routes. Mol Ecol 24(2):374-388. https://doi.org/10.1111/mec.13040

Haight KL, Tschinkel WR (2003) Patterns of venom synthesis and use in the fire ant Solenopsis invicta. Toxicon 42(6):673-682

Heller B, Sundermann B, Buschmann H, Drexler H-J, You J, Holzgrabe U, Heller E, Oehme G (2002) Photocatalyzed [2+2+2] cycloaddition of nitriels with acetylene: an effective method for the synthesis of 2-pyridines under mild conditions. J Org Chem 67:44144422. https://doi.org/10.1021/jo011032n

Lai LC et al (2009) Secretion profiles of venom alkaloids in Solenopsis geminata (Hymenoptera: Formicidae) in Taiwan. Environ Entomol 38(3):879-884. https://doi.org/10.1603/022.038.0342

Leclercq S, Braekman JC, Daloze D, Pasteels JM, Vander Meer RK (1996) Biosynthesis of the solenopsins, venom alkaloids of the 
fire ants. Naturwissenschaften 83(5):222-225. https://doi.org/10. 1007/BF01143328

MacConnell JG, Blum MS et al (1971) The chemistry of fire ant venom. Tetrahedron 27(6):1129-1139. https://doi.org/10.1016/ S0040-4020(01)90860-9

MacConnell JG, Blum MS et al (1976) Fire ant venoms: chemotaxonomic correlations with alkaloidal compositions. Toxicon 14(1):69-78. https://doi.org/10.1016/0041-0101(76)90122-7

Okuma K, Sakai O, Shioji K (2003) Wittig reaction by using DBU as a base. Bull Chem Soc Jpn 76:1675-1676. https://doi.org/10. 1246/bcsj.76.1675

Pitts JP, Camacho GP, Gotzek D, Mchugh JV, Ross KG (2018) Revision of the fire ants of the Solenopsis saevissima species-group (Hymenoptera: Formicidae). Proc Entomol Soc Wash 120(2):308411. https://doi.org/10.4289/0013-8797.120.2.308

Proffe E, Linke H-W (1960) Pyridyl-(2)-alkanole und pyridyl-(2)athylene. Chem Ber 93:2591-2603
Shi QH, Hu L et al (2015) Workers and alate queens of Solenopsis geminata share qualitatively similar but quantitatively different venom alkaloid chemistry. Front Ecol Evol 3(76):9. https://doi. org/10.3389/fevo.2015.00076

Vander Meer RK, Lofgren CS, et al (1985) Biochemical evidence for hybridization in fire ants. Fla Entomol 68: 501-506. http://www. fcla.edu/FlaEnt/

Wetterer JK (2011) Worldwide spread of the tropical fire ant, Solenopsis geminata (Hymenoptera: Formicidae). Myrmecolo News $14: 21-35$

Publisher's note Springer Nature remains neutral with regard to jurisdictional claims in published maps and institutional affiliations. 